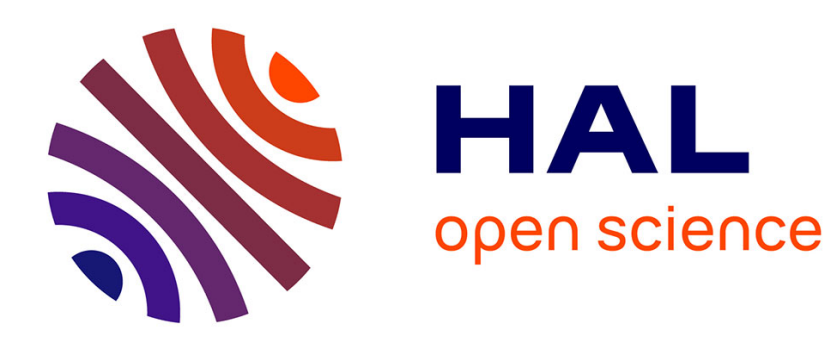

\title{
LE CYCLE SEXUEL DU BLAIREAU MÂLE (MELES MELES L.)
}

Marie-Claude Audy-Relexans

\section{To cite this version:}

Marie-Claude Audy-Relexans. LE CYCLE SEXUEL DU BLAIREAU MÂLE (MELES MELES L.).

Annales de biologie animale, biochimie, biophysique, 1972, 12 (3), pp.355-366. hal-00896719

\section{HAL Id: hal-00896719 \\ https://hal.science/hal-00896719}

Submitted on 1 Jan 1972

HAL is a multi-disciplinary open access archive for the deposit and dissemination of scientific research documents, whether they are published or not. The documents may come from teaching and research institutions in France or abroad, or from public or private research centers.
L'archive ouverte pluridisciplinaire HAL, est destinée au dépôt et à la diffusion de documents scientifiques de niveau recherche, publiés ou non, émanant des établissements d'enseignement et de recherche français ou étrangers, des laboratoires publics ou privés. 
Ann. Biol. anim. Bioch. Biophys., I972, 12 (3), 355-366.

\title{
LE GYCLE SEXUEL DU BLAIREAU MÂLE (MELES MELES L.)
}

\author{
Marie-Claude AUDY-RELEXANS ( ${ }^{1}$ ) \\ Centre d'Études biologiques des Animaux sauvages, C. N. R. S., \\ 79 - Villiers-en-Bois
}

\section{RÉSUMÉ}

Le Blaireau européen mâle présente des variations saisonnières des différents constituants de son appareil génital.

Le poids du testicule et de l'épididyme, la taille et le poids du baculum, les diamètres des tubes séminifères, la taille des cellules interstitielles et la hauteur de l'épithélium de l'épididyme, présentent des variations parallèles dont le maximum se rencontre aux mois de janvier-février qui correspondent aux mois du rapprochement sexuel fécondant. La spermatogenèse persiste tout au long de l'année.

Cependant, si les différences des structures histologiques entre des testicules prélevés à différentes périodes du cycle ne sont pas très apparentes, le nombre des cellules de la lignée germinale, par contre, subit une importante augmentation lors de la période d'activité maximale.

\section{INTRODUCTION}

Le Blaireau européen (Meles meles L.) est un Mammifère appartenant à l'ordre des Fissipèdes. Il fait partie, avec un grand nombre d'autres animaux sauvages, de la famille des Mustelidae et de la sous-famille des Melinae. C'est un animal omnivore, fouisseur, à l'activité essentiellement nocturne, que l'on trouve en assez grand nombre dans nos régions.

Le développement embryonnaire dans cette espèce est discontinu, et la femelle ou Blairelle présente le phénomène d'ovo-implantation différée qui a été découvert par HERBST en I873 et par FrIES en I880, observé par PRELL en I930 et Fischer en I93I, et longuement étudié par Canivenc (I960) et Bonnin-LaFFargue (I964).

Ces derniers auteurs ont montré que la mise bas se produit au mois de février.

(1) Attachée de Recherches au C. N. R. S. Lab. d'Endocrinologie Expérimentale UER Sciences Médicales II Université Bordeaux II. 
La femelle entre en œstrus sitôt après; la fécondation a lieu et le développement se poursuit jusqu'au stade blastocytaire, mais l'œuf restera libre, à ce stade, dans les voies génitales de la femelle pendant Io mois puisqu'il ne s'implantera qu'au mois de décembre.

L'accouplement et la fécondation effective montrent que le Blaireau mâle est en activité génitale optimum au mois de février. Mais cette activité se manifeste-t-elle uniformément tout au long de l'année ou existe-t-il un cycle net comme chez beaucoup de Mammifères sauvages? Il s'agit donc d'établir chez le Blaireau les caractéristiques de ce cycle génital.

C'est la raison pour laquelle une étude morphologique et histologique de l'appareil génital mâle et des glandes annexes s'impose.

\section{MATÉRIEL, E'T MÉTHODE}

Dans cette étude, nous utilisons 223 blaireaux mâles, adultes et impubères. Les animaux capturés vivants par déterrage sont envoyés immédiatement à notre laboratoire. L'animal préalablement pesé est sacrifié par une injection intra cardiaque de nembutal, les pièces sont prélevées immédiatement, disséquées, pesées et fixées dans du liquide de Bouin. Les mesures histologiques sont effectuées à l'aide d'un micromètre oculaire sur des coupes de $5 \mu$ d'épaisseur, colorées soit au PAS-Hématoxyline, soit au Bleu Masson.

Les estimations des différentes catégories cellulaires de la lignée germinale sont réalisées à partir des formules d'ABERCombie (1946).

$$
N_{R}=N_{O} \times \frac{E}{E+2 \sqrt{\left(\frac{D M}{2}\right)^{2}-\left(\frac{D i}{2}\right)^{2}}}
$$

$\mathrm{N}_{\mathbf{R}}$ : Nombre réel d'une catégorie de cellules germinales dans une section.

$N_{0}$ : Nombre observé compté directement dans les tubes séminifères à un stade choisi (stades d'ORTAVANT, I958).

E : Épaisseur de la coupe.

DM : Diamètre nucléaire moyen d'une catégorie cellulaire.

Di : Diamètre nucléaire inférieur d'une catégorie cellulaire.

Pour le calcul du nombre total d'une catégorie cellulaire dans un testicule, nous appliquons la formule modifiée par Martinet (1963).

$$
\mathrm{N}=\frac{\text { Volume testiculaire } \times \text { Nombre d'une catégorie cellulaire par section de tube }}{\text { Surface section des tubes séminifères } \times \text { épaisseur de section }}
$$

Ce calcul permet des comparaisons relatives entre les éléments germinaux de testicules prélevés à des époques différentes de l'année et non une étude quantitative exacte du nombre de ces éléments.

Les baculums ou os péniens recueillis sont étudiés après dessiccation, soigneusement pesés et étiquettés. Ils sont particulièrement utiles pour déterminer l'état de maturité sexuelle des animaux.

\section{RÉSULTATS}

Dans une population de blaireaux à l'état sauvage, le prélèvement par capture d'individus intéresse toutes les classes d'âge de cette population. Aussi, il paraît indispensable de savoir si l'on a affaire à des sujets matures ou immatures. Le Blaireau étant un animal dont le poids corporel peut varier du simple au double au cours 
de l'année, il n'est pas possible de retenir ce seul critère et l'on doit en rechercher d'autres pour reconnaître un animal adulte d'un impubère. Les résultats consignés ci-dessous en sont une démonstration.

\section{Poids du corps}

Le poids corporel des animaux est important et peut atteindre un maximum de 16 kilogrammes, mais le poids d'un même individu varie au cours de l'année de façon très sensible et selon un cycle été-hiver assez net (M.-C. REL.EXArrs et R. CANIVENC, I967).

A l'entrée de l'hiver en effet, l'animal emmagasine une grande quantité de réserves graisseuses et c'est toujours aux mois de novembre et décembre que les poids sont les plus importants, alors qu'au mois de mars ou avril, les valeurs pondérales sont les plus basses (6 à 8 kilogrammes).

Cependant, le poids de certains individus atteint une valeur très voisine de celle de la moyenne maximum à n'importe quelle époque de l'année; la figure I représente donc les variations des valeurs moyennes des poids en fonction du mois de l'année et non les valeurs individuelles de ces poids.

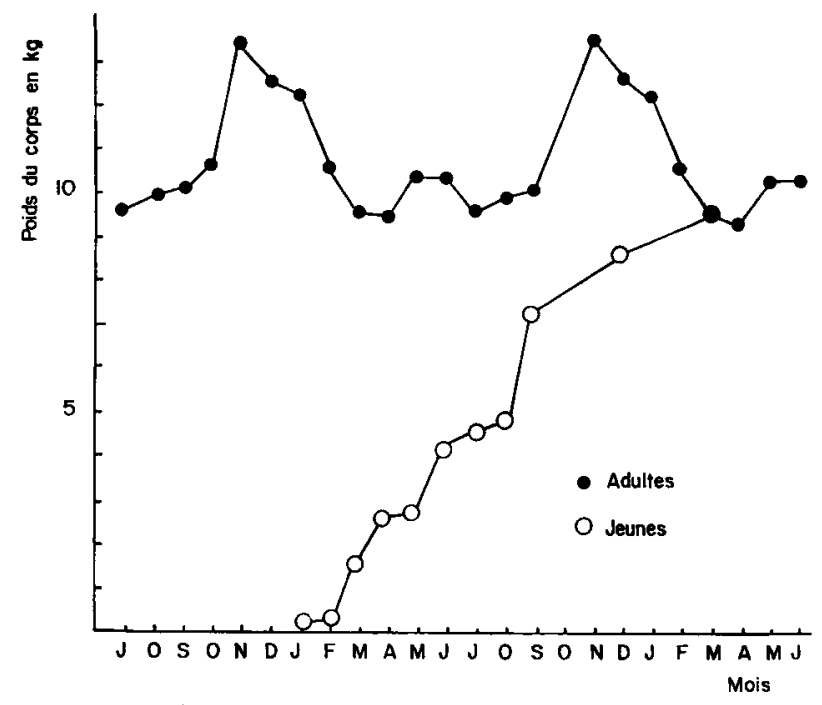

FIG. I. - Evolution du poids du corps en fonction de la saison

La croissance pondérale du jeune (fig. I) se fait rapidement et au bout d'un an, il n'est déjà plus possible de différencier un adulte d'un jeune en se basant seulement sur sa taille ou son poids. Il est donc nécessaire de disposer d'autres critères. A ce point de vue, la taille et le poids du baculum apportent des documents précieux.

\section{Baculum}

La présence d'un os pénien ou baculum a attiré l'attention de nombreux auteurs et ceci dans des groupes zoologiques très variés. Cet os pénien est signalé dans de 
nombreux groupes : Fissipèdes, Pinnipèdes, Rongeurs, Insectivores, Dermoptères, Chiroptères et Primates (P.-P. GRassÉ, I955).

Lorsqu'il existe, on utilise les variations de son poids ou de sa taille comme critère de l'âge de l'animal.

L'anatomie $d u$ baculum de nombreux Mustelidae a été décrite par LoNG (I969), en particulier celui du Blaireau américain (Taxidea taxus). Le baculum du Blaireau européen est de forme allongée et peut atteindre une longueur de 8,5 centimètres, plus ou moins incurvé mais toujours très légèrement; il possède une ouverture à son extrémité distale. Son poids atteint une valeur maximale de $I, 9$ grammes et au cours de la croissance son diamètre postérieur augmente jusqu'à atteindre une valeur de I centimètre. (fig. 2 ).

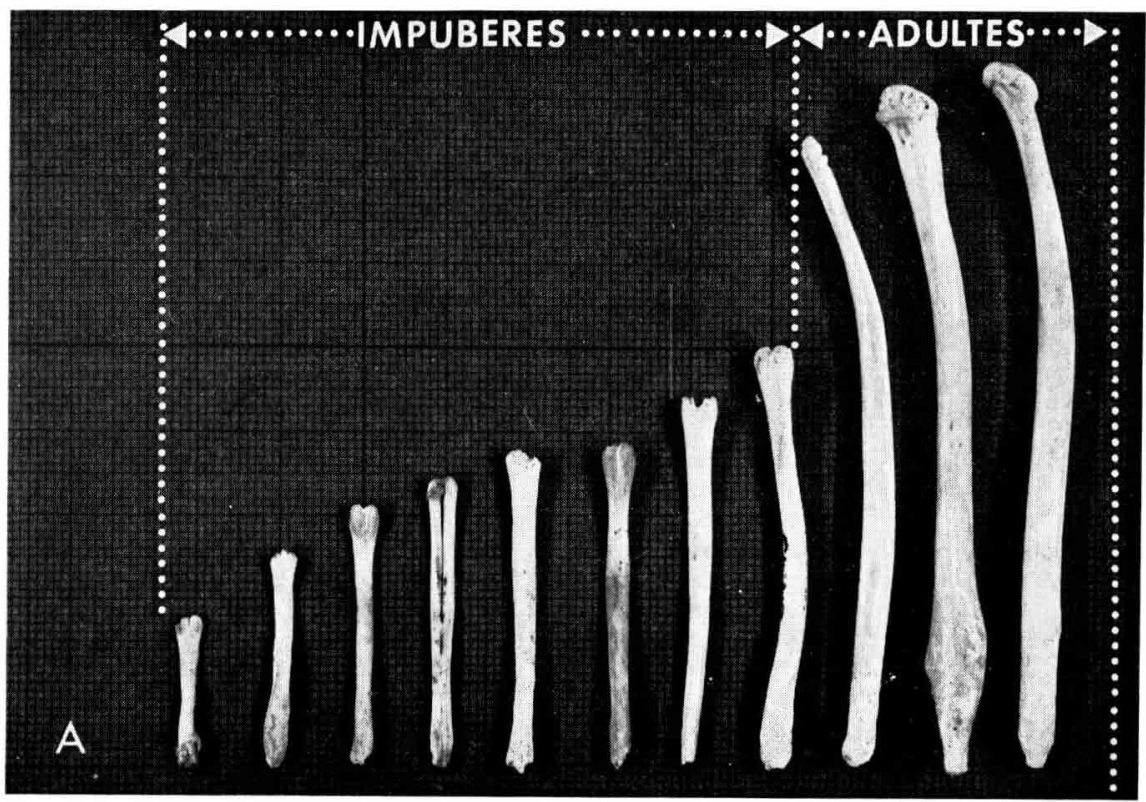

FIG. 2 Évolution de la taille et de la forme du baculum du Blaireau européen (Meles meles L.) en fonction de l'âge

\section{a) Variation de la taille}

La longueur du baculum s'accroît régulièrement au cours du développement, de l'animal. Dès la première année, la baculum atteint le maximum de longueur, et cette valeur ne varie ensuite que très peu, ce qui se traduit par un aplatissement de la courbe. (fig. 3 ).

L'étude de 6r baculums recueillis, montre que la longueur permet de déterminer 1'âge mais seulement au cours de la première année de la vie de l'animal. Après cette période, il n'est plus possible d'établir l'âge avec certitude.

b) Variation du poids.

Le poids du baculum s'accroît d'abord assez lentement pendant les six premiers mois qui suivent la naissance (février à septembre) beaucoup plus rapidement au 


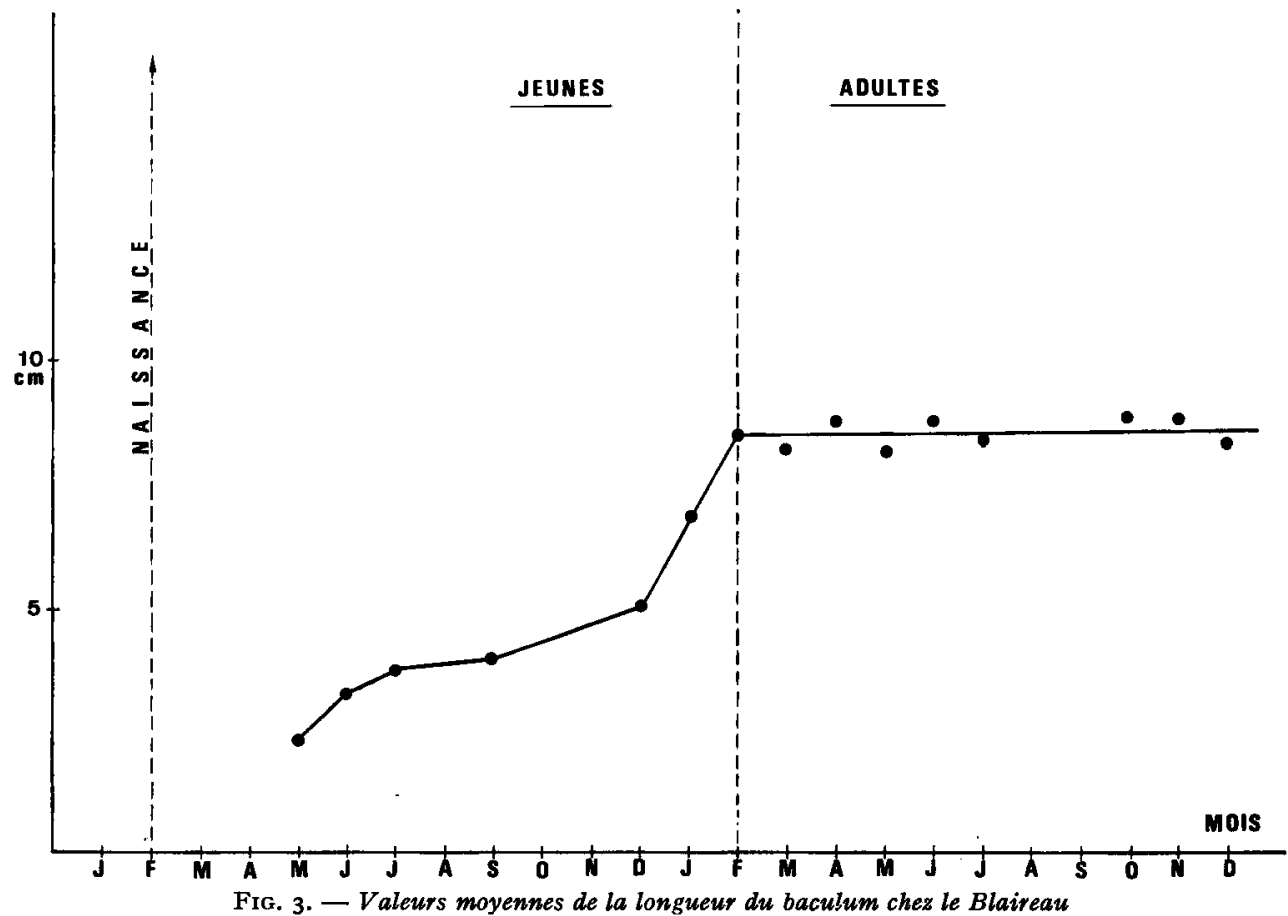

FIG. 3. - Valeurs moyennes de la longueur du baculum chez le Blaireau

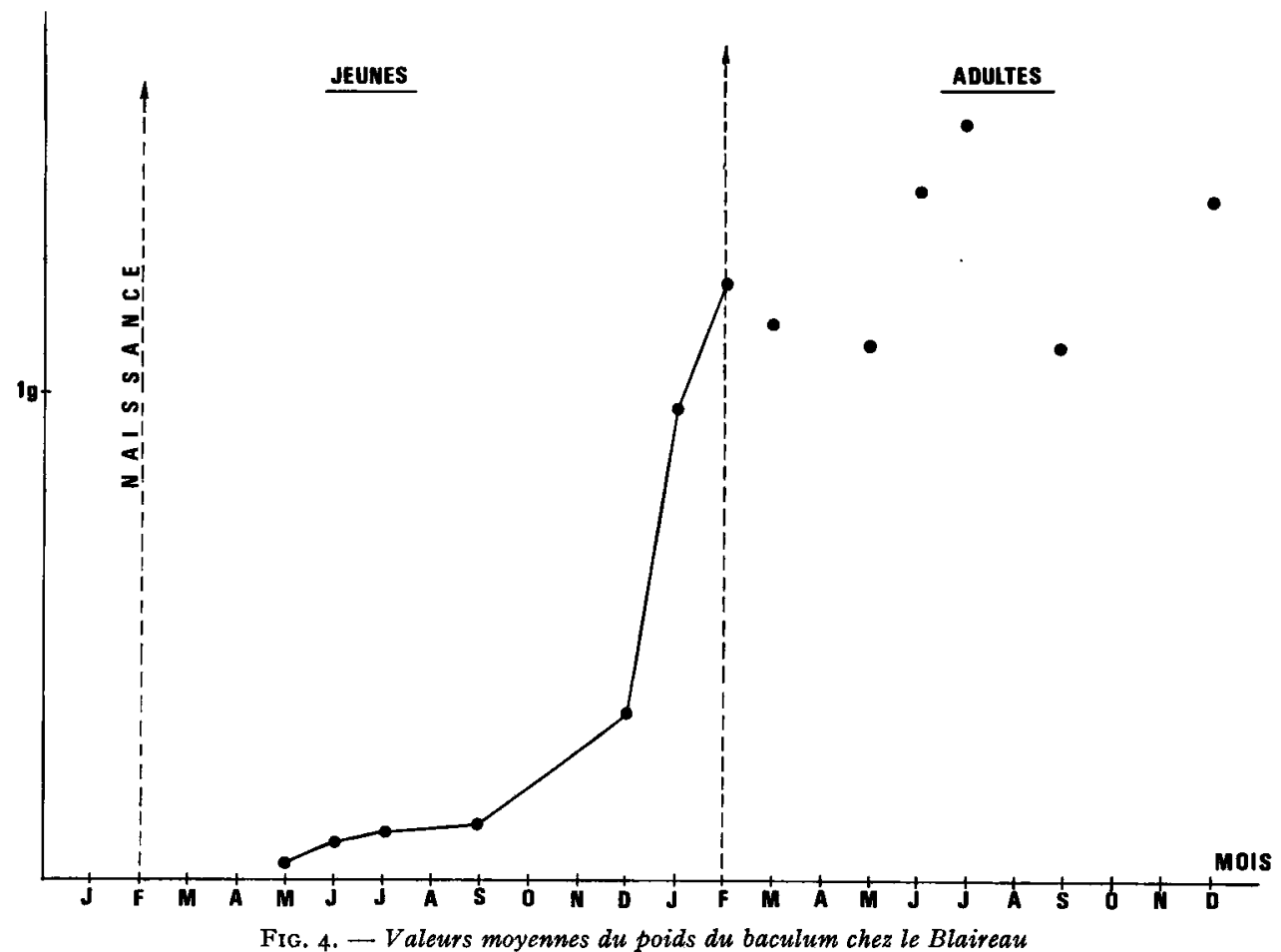


cours des mois suivants (septembre à février) et atteint une valeur voisine de I $\mathrm{g}$ dès la première année. Ensuite, au cours de la vie de 1'animal, le poids est variable avec l'âge et selon les individus et pour cette raison la figure 4 ne présente pas un plateau proprement dit mais des points disséminés.

L'évolution différente du poids et de la taille du baculum avec l'âge résulte donc de son épaississement. Pour utiliser ce critère pondéral, il faudrait posséder un élevage de blaireaux afin d'établir une courbe étalon à partir d'animaux d'âge connu. Ceci nous est impossible puisque, jusqu'à présent, nous n'avons pas obtenu la reproduction du Blaireau en captivité. C'est ce que SANDERSON (I96I) a réalisé chez le Raton laveur (Procyon lotor). Toutefois, bien qu'il ait dans ses séries des animaux d'âge connu permettant d'établir une courbe étalon de la variation de la longueur, il utilise plusieurs critères pour déterminer l'âge de ses animaux (poids du corps - étude des sutures épiphysaires).

WaLTon (Ig68) chez le Putois (Putorius putorius L.) trace une courbe de croissance des baculums, mais il arrive également à la conclusion que sans élevage on ne peut connaître 1'âge exact des animaux à 1'aide du baculum, tout au plus, peut-on affirmer si l'animal est jeune ou âgé.

\section{Variations pondérales du testicule et de l'épididyme}

L'étude pondérale du testicule et celle de 1'épididyme au cours de l'année fournissent une première appréciation de l'existence d'un cycle d'activité génitale mâle. En traçant une courbe des valeurs moyennes mensuelles des poids testicu-

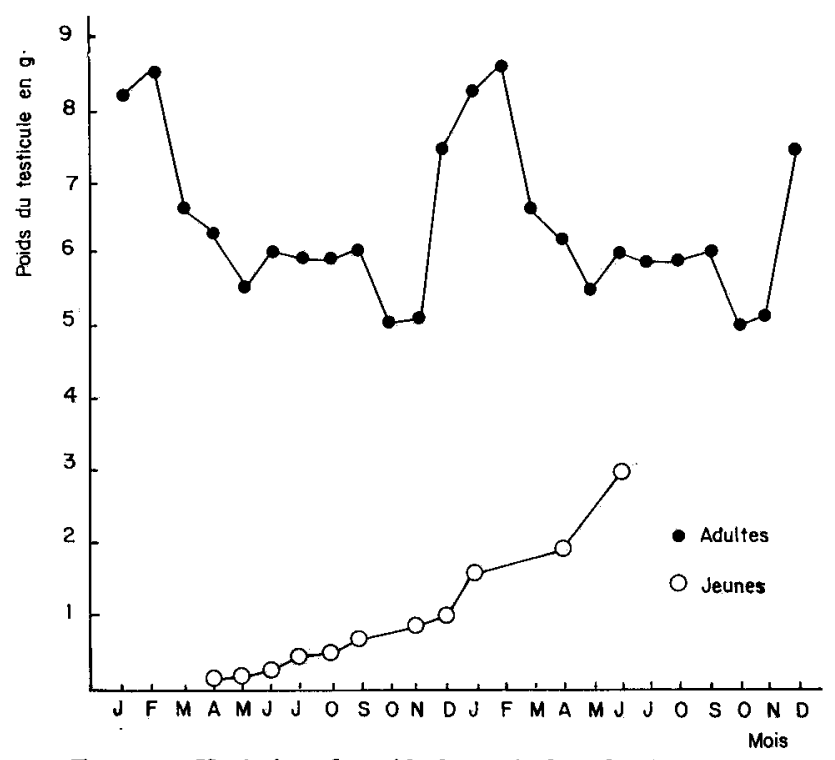

Fig. 5. - Variations du poids des testicules selon la saison

laires et des poids épididymaires, on observe un maximum aux mois de janvierfévrier, période d'accouplement et de fécondation (M.-C. RELEXANs et R. CANIVENC I967). 


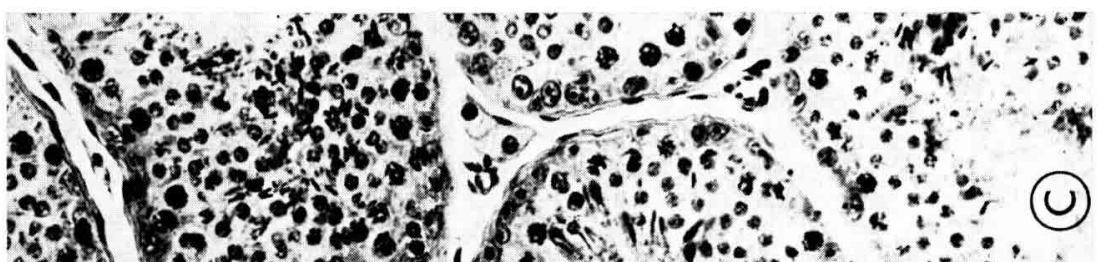
3 (1)

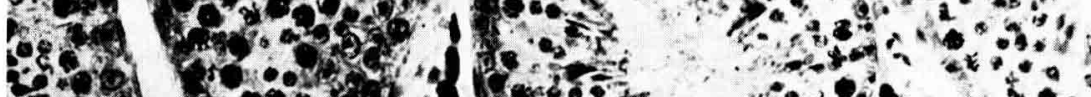
2. 5. 6.5

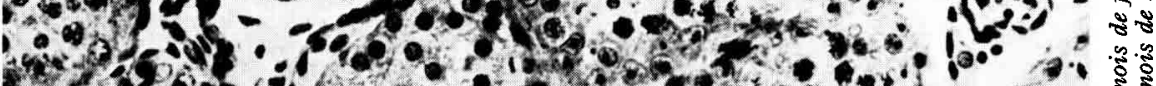

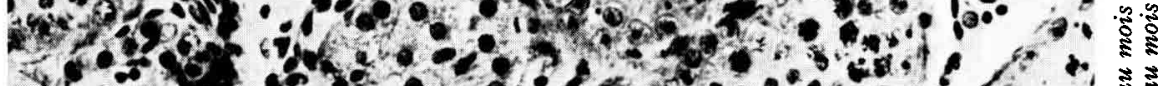

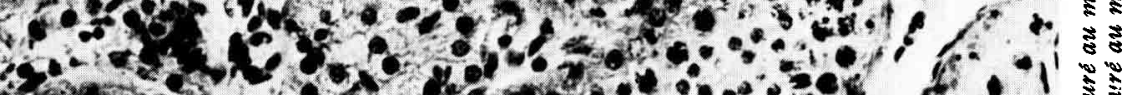

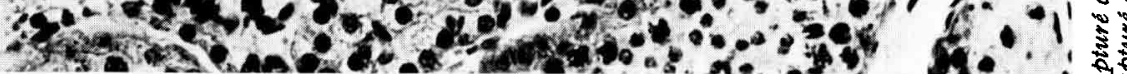

उ

空

홀

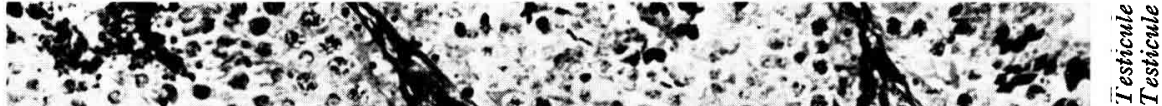
(a)

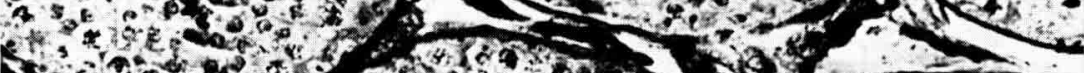

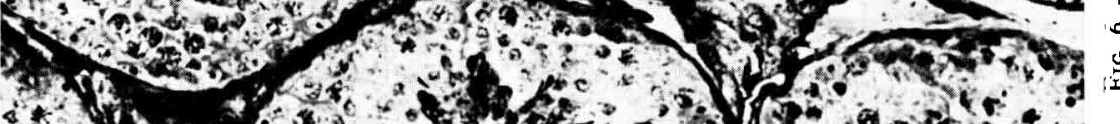

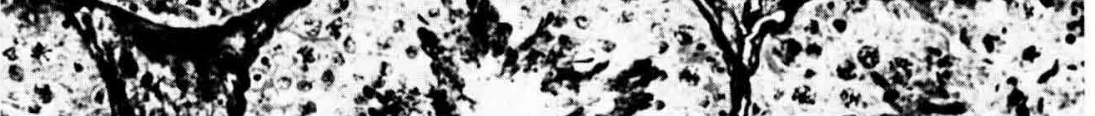

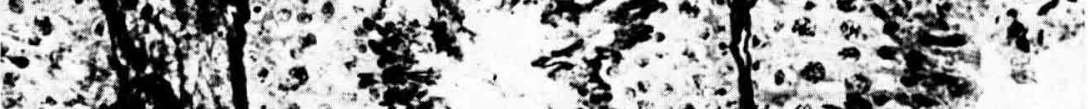

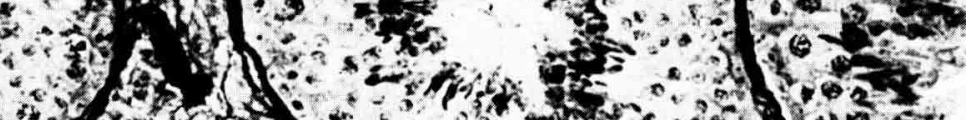

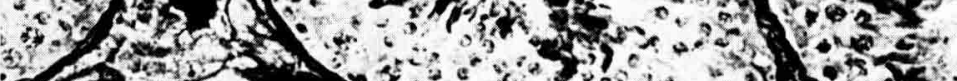

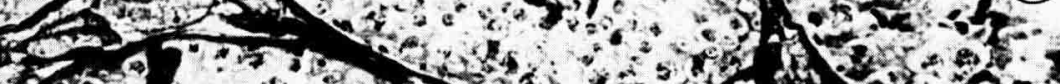

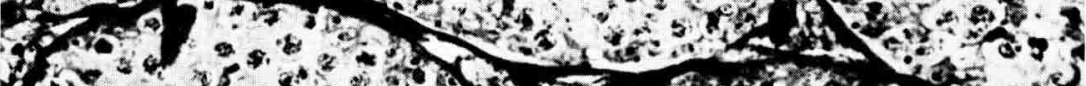

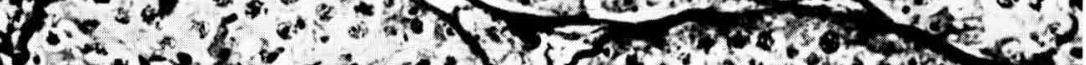

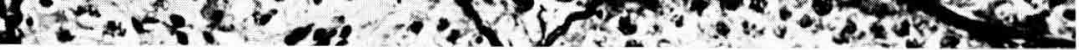


La croissance pondérale du testicule au début de la vie de l'animal (fig. 5) évolue lentement; au bout de la première année (au mois de mars), le testicule de certains animaux présente déjà des tubes séminifères avec une lignée germinale complète, mais c'est au cours de la deuxième année que la plupart d'entre eux acquerront la totalité des stades de la lignée germinale.

Il est donc possible, en utilisant les critères de poids corporels et testiculaires, la longueur et le poids du baculum, de déterminer l'âge de l'animal ou du moins de savoir si l'animal est un jeune ou un adulte, mais au-delà de la période de maturité sexuelle, il n'est plus possible de connaître par ces seuls critères, l'âge de l'animal.

\section{Étude histologique.}

La présence de spermatozoïdes dans l'épididyme tout au long de l'année, indique que la spermatogenèse demeure active. Pour préciser l'activité du testicule et de l'épididyme, nous avons effectué une étude comparée en prélevant les organes au moment où les poids sont maximums, en février, ou minimums, en juillet (fig. 6).

Nous n'observons pas alors de différence qualitative notable des images histologiques de testicules prélevés à ces différentes périodes de l'année, tous les stades de la spermatogenèse $y$ sont reconnaissables.

Mais l'étude du nombre des cellules germinales à deux périodes de l'année (hiverété) montre des différences importantes, résumées dans le tableau ci-dessous.

TABLEAU I

Nombre total de cellules dans un testicule

\begin{tabular}{|c|c|c|}
\hline \multirow{2}{*}{ Catégorie cellulaire } & \multicolumn{2}{|c|}{ Nombres $\times 10^{6}$} \\
\hline & Repos & Activité \\
\hline Sertoli . . . . . . . . . & 251 & 333 \\
\hline Spermatogonies ...... & 66 & 88 \\
\hline Leptotènes ....... & 520 & 726 \\
\hline Pachytènes . . . . . . . & 559 & 726 \\
\hline Spermatides . . & 1971 & 2685 \\
\hline
\end{tabular}

Les éléments de la lignée germinale subissent une très forte augmentation de leur nombre lors de la période d'accouplement (hiver). Ce phénomène intéresse tous les stades de la spermatogenèse et il existe un accroissement parallèle du nombre de chaque catégorie de cellules germinales.

\section{Étude histométrique}

Des mensurations des différents paramètres testiculaires sont effectuées sur des coupes minces. Nous avons étudié spécialement le diamètre des tubes séminifères et la taille des cellules interstitielles ainsi que la hauteur de l'épithélium de l'épididyme : 
a) Variation du diamètre des tubes séminifères.

Chez le Blaireau, le diamètre des tubes séminifères subit une variation assez importante au cours de l'année, puisqu'il passe d'une valeur de $I 98 \mu$ en période de reproduction à une valeur de $168 \mu$ en été. Cependant, à l'intérieur du tube séminifère, nous n'observons pas de variation de la lignée germinale comme cela est le cas chez d'autres espèces de Mustelidae (Martres, Blaireau américain, etc.). Tout au plus existe-t-il chez le Blaireau, une différence de densité de ces éléments. (fig 7.)

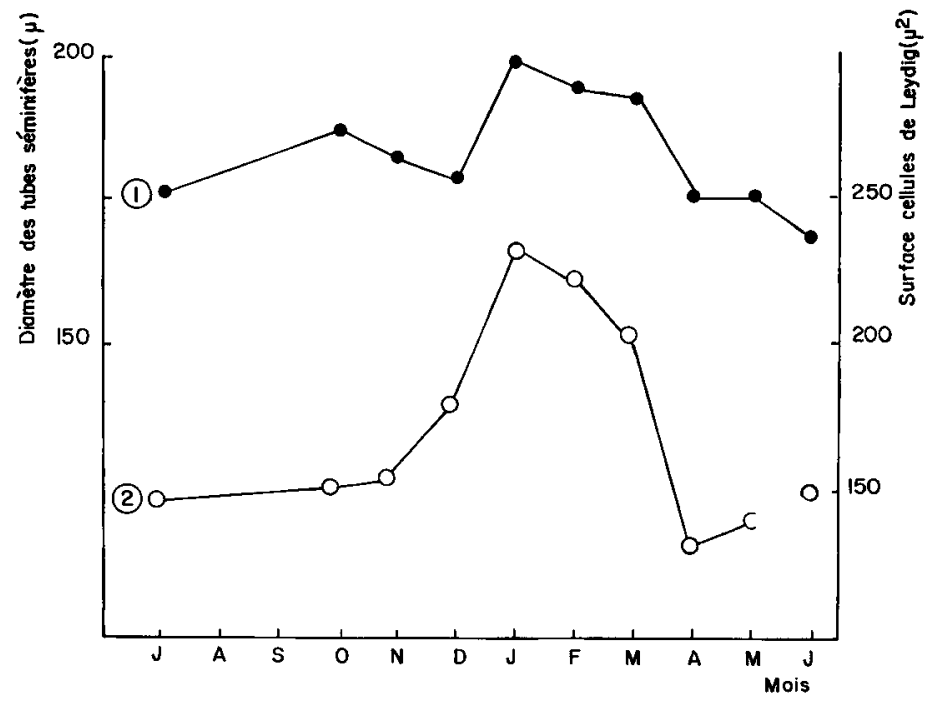

Fig. 7. - Variation du diamètre des tubes séminifères et de la surface des cellules de Leydig selon la saison

b) Variation de la taille des cellules interstitielles.

Les surfaces des cellules de Leydig sont calculées en les assimilant à des ellipsoïdes et en utilisant la formule $\mathrm{S}=\frac{\pi \mathrm{D} d}{4}$ où $\mathrm{D}$ est la valeur moyenne du plus grand diamètre et $d$ la valeur moyenne du plus petit diamètre.

Ces surfaces cellulaires ont une valeur maximale en janvier-février, suivie d'une diminution assez importante à la fin de la période de reproduction, au mois d'avril (fig. 7).

\section{c) Variation de la hauteur des cellules de l'épithélium de l'épididyme.}

Il existe une concordance parfaite entre les variations du poids et de la hauteur de l'épithélium de l'épididyme. La hauteur maximale des cellules épithéliales est de $42 \mu$ au mois de janvier et la hauteur minimale de $30 \mu$ au mois d'octobre, ce qui correspond parfaitement aux variations des autres éléments de l'appareil génital. (fig. 8)

En conclusion, l'étude pondérale et histométrique des différents constituants testiculaires met en évidence une série de variations qui sont parallèles entre elles. Même faibles, ces variations ne sont pas dues au hasard, les observations ayant porté sur un grand nombre d'animaux et sur plusieurs cycles annuels. De plus, les variations importantes de la quantité des éléments germinaux permettent de conclure 
à l'existence d'un cycle génital avec une période d'activité maximale en hiver aux mois de janvier-février et une période d'activité minimale en été aux mois de juilletaoût.

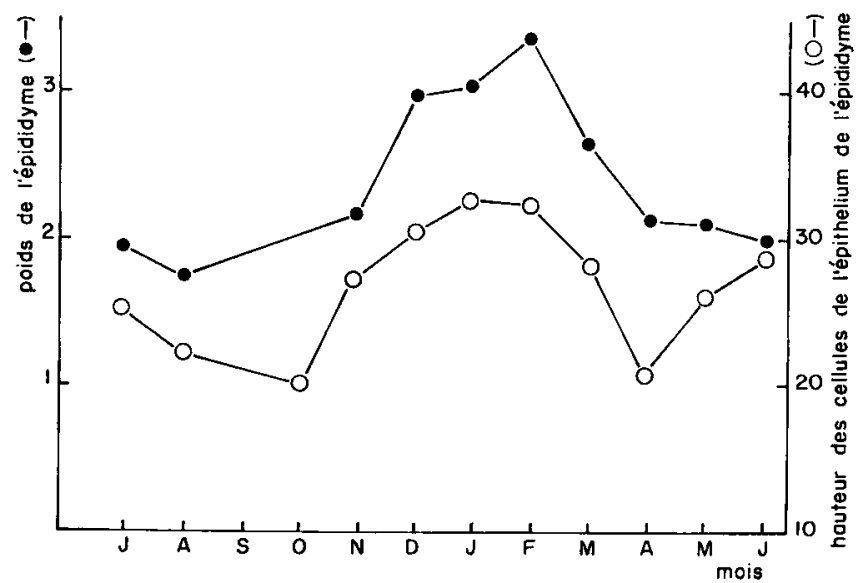

Fig. 8. - Variation du poids de l'épididyme et de la hauteur de l'épithélium selon les saisons

\section{DISCUSSION DES RÉSULTATS}

Chez les Mammifères sauvages, il est fréquent de rencontrer des variations très notables des paramètres génitaux avec des périodes de repos et d'activité testiculaires fort nettes.

Certains animaux présentent, en effet, un arrêt complet de la spermatogenèse à une ou plusieurs périodes de l'année. Dans ces cas, les valeurs histométriques des constituants de l'appareil génital subissent des variations très importantes. C'est le cas du Skung (Spilogale putorius) décrit par MEAD en Ig66. MEAD signale la disparition de la lignée spermatogénétique au cours du repos sexuel hivernal. Le poids des testicules est de $247 \mathrm{mg}$ en septembre ; cette valeur subit ensuite une augmentation considérable, puisqu'au mois d'avril elle est de $5400 \mathrm{mg}$. Les courbes de la variation des diamètres des tubes séminifères et du noyau des cellules de Leydig présentent des variations parallèles.

De même chez d'autres Mustelidae, le diamètre des tubes séminifères de la Martre varie de $60 \mu$ en phase de repos (au mois de décembre) à $200 \mu$ en phase d'activité (juillet). (RELEXANS et CANIVENC, sous presse). Dans la même espèce, il existe une très grande variation des poids testiculaires entre la période de repos (170 $\mathrm{mg}$ ) et la période d'activité ( $\mathrm{I} 900 \mathrm{mg}$ ) (R. CANIVENC, M. Bonnin-LAFFarGuE, M.-C. RELEXANS, I968).

Le Blaireau américain (Taxidea taxus) est d'un très grand intérêt pour cette étude, car il est zoologiquement très proche de Meles meles L. et présente avec ce dernier des différences notables quant à son activité génitale. C'est ainsi que WRIGH'T (r969) signale l'existence d'un cycle génital net, mais dont l'activité testiculaire maximale est située en été (juin-juillet-août), alors que chez le Blaireau européen, ce maximum a lieu en janvier; d'autre part, WRIGHT propose des images 
histologiques des testicules de ses animaux où l'on constate, au cours du repos sexuel, l'existence d'une dégénérescence de la lignée germinale très importante, comparable à celle observée chez la Martre. Nous ne retrouvons pas chez le Blaireau européen des images identiques.

D'autres animaux au contraire présentent une simple fluctuation de leur activité testiculaire sans arrêt complet de la spermatogenèse. C'est le cas du Campagnol (Microtus arvalis). En prenant soin de n'étudier que des mâles pubères de même âge ( 2 à 4 mois) L. MarTinet, en I968, montre que même si le poids des testicules est minimum d'octobre à février, le nombre de spermatogonies varie peu mais que les rendements leptotènes/gonies, pachytènes/leptotènes et spermatides/pachytènes passent par un maximum d'avril à juillet.

Chez le Blaireau européen, NEAL et HARRISSON en I958, avaient déjà représenté une courbe des variations des diamètres des tubes séminifères au cours de l'année avec une valeur maximale de $185 \mu$ en mars et une valeur minimale de $135 \mu$ en février. Ces auteurs indiquaient également l'existence "d'un déclin de l'activité " au mois de septembre, mais sans préciser le mođe de diminution de cette activité.

Nous ne retrouvons pas exactement les mêmes résultats sans doute à cause du plus grand nombre d'animaux dont nous avons disposé, à moins qu'il ne s'agisse d'une particularité géographique.

En effet, les animaux adultes que nous avons observés présentent des variations du poids testiculaire avec une valeur maximale aux mois de janvier-février. D'autre part, le diamètre des tubes séminifères varie également mais sans qu'il y ait des écarts considérables entre les différentes périodes de l'année. Nous avons, en outre, observé une spermatogenèse complète tout au long de l'année et une persistance des réserves épididymaires. Enfin, par des comptages des différentes catégories cellulaires de la lignée germinale à deux périodes de l'année (l'une correspondant au maximum des variations des différents constituants testiculaires, l'autre au minimum), nous constatons une différence notable de leur quantité, avec une valeur beaucoup plus importante aux mois de janvier-février (période de la reproduction) qu'aux mois de juillet-septembre. Il existe donc bien, chez, le Blaireau, un cycle de l'activité génitale mâle se traduisant par une production quantitativement différente des éléments germinaux.

Reçu pour publication en janvier 1972.

\section{REMERCIEMENTS}

Nous remercions le Laboratoire de Physiologie de la Reproduction de l'I.N.R.A. à Nouzilly de son accueil et en particulier Mme de Reviers qui nous a initié au comptage des catégories cellulaires.

\section{SUMMARY}

\section{THE SEX CYCLE OF THE MALE BADGER (MELES MELES L.)}

Various parts of the genital system of the European male badger have seasonal variation.

Testicle and epididymis weight, size and weight of the baculum, seminal tube diameter, interstitial cell size, and the height of epididymis epithelium have parallel variation, the maximum 
of which occurs in the months of January-February. These months correspond to the mating period. Spermatogenesis occurs all year long.

However, while differences in histological structure are not very apparent in testicles sampled at various periods during the cycle, the number of germinal cells greatly increases during the period of maximum activity.

\section{RÉFÉRENCES BIBLIOGRAPHIQUES}

Abercombie M., I946. Estimation of nuclear population from microtome sections. Anat. Rec., 94, 238-248.

Bonnin-Laffargue M., 1964. Contribution à l'étude de l'histophysiologie de l'appareil génital femelle du Blaireau européen (Meles meles L.). Thèse Doctorat ès Sciences, I73, Bordeaux

Canivenc R., r96o. L'ovo-implantation différée des animaux sauvages. Colloque sur les fonctions de nidation utérine et leurs troubles, 33-86, Masson Éd., Paris.

Canivenc R., Bonnin-Laffargue M. Relexans M. C., rg68. Cycles génitaux de quelques Mustellidés européens. In : Entretiens de Chizé, Physiologie n ${ }^{\circ} 1$, Éd. Masson.

Fischer E., I931. Early Stages in Embryology of the Badger. Verh. Anat. Ges., Iena, 40, 22.

Fries S. Uber die fortpflanzung von Meles taxus. Zool. Anz., 3, 486-492.

Grassê P. P., I955. Traité de Zoologie, Anatomie, Systématique, Biologie, 16, I 62 , Éd. Masson et Cie.

Herbst G., 1873. Wann ranzt der Dachs? Ans, der. Walde.

Long Ch. A., 1969. Gross Morphology of the penis in seven species of the Mustelidae. Mammalia, 33, I45-I6o.

Martinet L., 1963. Établissement de la spermatogenèse chez le Campagnol des champs (Microtus arvalis) en fonction de la durée quotidienne d'éclairement. Ann. Biol. anim. Bioch. Biophys., 3 (4), $343-352$.

Martinet L., 1968. Cycle saisonnier de reproduction du Campagnol des champs (Microtus arvalis)In : Entretiens de Chizé, Série Physiologie no 1, Éd. Masson.

MEAd R. A., 1966. Reproduction in the spotted skund (Spilogale putorius). These University of California, II9.

Neal G. E., Harrisson R. J., I958. Reproduction in the European Badger (Meles meles L.). Trans. Roy. Zool. Soc. Lond., 29, 67-130.

Ortavant R., 1958. Le cycle spermatogénétique chez le Bélier. Th. Doctorat ès Sciences, Paris, p. I27.

Prell M., I930. Period of gestation in Mustelids. Zool. Anz., Leipzig, 87, 273-283.

Relexans M.-C., Canivenc R., I967. Évolution pondérale du testicule du Blaireau européen (Meles meles L.) au cours du cycle génital annuel. C. R. Soc. Biol., 161, no $3,600-603$.

Sanderson G. C., I96r. The reproductive cycle and related phenomena in the racoon. These University of Illinois, I58.

Walton K. C., I968. The baculum as an age indicator in the pole cat (Putorius putorius). J. Zool. Lond., 156, 533-536.

WRIGHT P. L., I969. Reproductive cycle of the male american badger (Taxidea taxus). J. Reproduc. Fert., Suppl. 6, 435-46. 